Molecular Physics

Vol. 00, No. 00, DD Month 201x, 1-22

\title{
Bonding Description of the Harpoon Mechanism ${ }^{\dagger}$
}

\author{
Mauricio Rodríguez-Mayorga ${ }^{a, b}$, Eloy Ramos-Cordoba $^{a}$, Pedro Salvador $^{b}$, \\ Miquel Solà ${ }^{b}$ and Eduard Matito ${ }^{a, c, *}$
}

Faculty of Chemistry, University of the Basque Country UPV/EHU, and Donostia

International Physics Center (DIPC). P.K. 1072, 20080 Donostia, Euskadi, Spain

${ }^{b}$ Institut de Química Computacional i Catàlisi (IQCC) and Departament de

Química, Univ. Girona, Campus de Montilivi s/n, Girona, Spain.

${ }^{c}$ IKERBASQUE, Basque Foundation for Science, 48011 Bilbao, Spain

(Received 00 Month 201x; In final form 00 Month 201x)

The lowest-lying states of $\mathrm{LiH}$ have been widely used to develop and calibrate many different methods in quantum mechanics. In this paper we show that the electron-transfer processes occurring in these two states are a difficult test for chemical bonding descriptors and can be used to assess new bonding descriptors on its ability to recognize the harpoon mechanism. To this aim, we study the bond formation mechanism in a series of diatomic molecules. In all studied electron-reorganization mechanisms, the maximal electron-transfer variation point along the bond formation path occurs when about half electron has been transferred from one atom to another. If the process takes places through a harpoon mechanism, this point of the reaction path coincides with the avoided crossing. The electron sharing indices and one-dimensional plots of the electron localization function and the Laplacian of the electron density along the molecular axis can be used to monitor the bond formation in diatomics and provide a distinction between the harpoon mechanism and a regular electron-reorganization process.

\section{Introduction}

The formation of molecules through the harpoon mechanism [1] occurs from the interaction of two fragments, one with a low ionization potential (IP) and another that has a large electron affinity (EA). The reactants approach each other and, at a certain distance, an electron from the fragment with low IP harpoons the fragment with large EA, giving rise to a rapid electron-transfer process that is triggered by the Coulomb attraction exerted by the two fragments. The interplay between the energy exchange due to the harpoon process and the Coulomb attraction determines the distance at which the electron transfer takes place. The harpoon mechanism was proposed by

\footnotetext{
$\dagger$ This paper is dedicated to Andreas Savin on the occasion of his $65^{\text {th }}$ birthday.

* Corresponding author. Email: ematito@gmail.com
} 
Michael Polanyi to explain the unusually large cross sections observed in the formation of alkali halides. Since the IP of the alkali metals is similar to the EA of halogens, the Coulomb attraction needed to favor the electron-transfer process is usually small for these species and, therefore, the electron transfer occurs at large separation of the reactants. On the other hand, if the molecule under consideration is an alkali hydride the process takes place at short distances because the EA of hydrogen is much smaller than the IP of alkalis.

Since the dissociation of ionic species in gas phase through the inverse harpoon mechanism usually involves the transition from ionic to neutral fragments in an electron-transfer process, the molecule is expected to change its electron sharing index (ESI) - commonly known as bond order - from a low (ionic) value at equilibrium to a larger value (when the electron transferred lies between the two fragments) that should decay as the fragments separate from each other. Ponec and coworkers 2 demonstrated that $\mathrm{LiH}$ and $\mathrm{BeH}$ show a maximum of the ESI in the vicinity of the avoided crossing between two adiabatic states. Interestingly, the ESI maximum was obtained using the quantum theory of atoms in molecules (QTAIM) definition of an atom, but it could not be reproduced using a Mulliken atomic partition. Some of us have demonstrated [3, 4] that many atomic partitions fail to attain this condition and suggested that the ability to reproduce this feature could be used as a criterion to assess the goodness of atomic partitions [3].

Thus far, the electron-transfer process has been also monitored using several quantities, including the dipole [5] and higher-order moments [6], the topological properties of the electron density [7, the electron localization function (ELF) [8, 9] and other descriptors of the electronic structure [2, 3, 10, 11. However, none of these methods has been able to actually follow the electron transferred as it jumps from one atom to the other. This sudden jump is what differentiates the harpoon mechanism from a regular electron-reorganization reaction and, therefore, its detection would provide a means to distinguish these mechanisms. Indeed, the three-dimensional pictures of the ELF do not reveal a separate basin for the electron until the electron has been already completely transferred to the other fragment $[9]$.

The goal of this work is analyzing the harpoon mechanism using a plethora of bonding descriptors to find which of these tools can be used to characterize the reaction. To this aim, we will analyze the dissociation of three ionic compounds that present the electron transfer at different interatomic separations and other small molecules, whose formation does not take place through a harpooning reaction. The analysis of these molecular systems shall shed some light on the abilities of some bonding tools, providing some interesting test 
cases that can be used in the development of new descriptors of the electronic structure of molecules.

\section{Methodology}

In this section we briefly review several bonding descriptors that will be used to study the harpoon mechanism. Let us assume a given partition of the molecular space into atomic regions that will be labeled $A, B, C, \ldots$ The integration of the electron density within an atomic region gives rise to the electron population:

$$
N(A)=\int_{A} \rho(\mathbf{r}) d \mathbf{r}
$$

where $\rho(\mathbf{r})$ is the electron density. Analogously, the pair density is needed to define pair populations,

$$
N(A, B)=\int_{A} \int_{B} \rho_{2}\left(\mathbf{r}_{1}, \mathbf{r}_{2}\right) d \mathbf{r}_{1} d \mathbf{r}_{2}
$$

which enter the expression of the electron sharing indices (ESI) or electron delocalization indices $[3,12,13$,

$$
\delta(A, B)=2[N(A) N(B)-N(A, B)]=-2 \operatorname{cov}(N(A), N(B))
$$

that are related to the covariance of the atomic populations. The ESI are a non-integer version of the classical concept of bond order [14] that provides a real value in accord with the number of electron pairs shared by two fragments.

The ELF requires the calculation of the Laplacian of the same-spin pair density functions [15 17] i.e.,

$$
\operatorname{ELF}(\mathbf{r})=\frac{1}{1+D(\mathbf{r})^{2}}
$$

where

$$
D(\mathbf{r})=\frac{\left.\nabla_{\mathbf{r}_{2}}^{2}\left(\rho_{2}^{\alpha \alpha}\left(\mathbf{r}, \mathbf{r}_{2}\right)+\rho_{2}^{\beta \beta}\left(\mathbf{r}, \mathbf{r}_{2}\right)\right)\right|_{\mathbf{r}_{2}=\mathbf{r}}}{2 c_{F} \rho^{8 / 3}(\mathbf{r})}
$$

with $c_{F}=\frac{3}{10}\left(3 \pi^{2}\right)^{2 / 3}$. The ELF measures the extent of electron localization, 
giving values close to zero for regions with highly delocalized electrons and large values in nuclear regions, lone pairs and bonding electron pairs [18. Similarly, negative values of the Laplacian of the electron density also determine molecular regions with localized electrons, sometimes providing an electronic picture similar to that of the ELF [19,20]. The three-dimensional representations of the ELF and the Laplacian have been repeatedly used in the literature to characterize all sorts of chemical bonds [20].

Information theory has been also used to characterize the bonding patterns and the electronic structure of molecular systems [21 23]. Among them, the most well known are Shannon entropies and Fisher information descriptors. The Shannon entropies are descriptors of the spread of the electronic density (in position or momentum space) in accord with the following expressions [24, 25

$$
\begin{gathered}
S_{\rho}=-\int \bar{\rho}(\mathbf{r}) \ln \bar{\rho}(\mathbf{r}) d \mathbf{r} \\
S_{\pi}=-\int \pi(\mathbf{p}) \ln \pi(\mathbf{p}) d \mathbf{p}
\end{gathered}
$$

where $\bar{\rho}(\mathbf{r})$ is the position space electronic density normalized to unity and the $\pi(\mathbf{p})$ is the momentum space electronic density also normalized to unity. The sum of both entropies, $S_{T}=S_{\rho}+S_{\pi}$, - known as total Shannon entropyhas a physical bound imposed by the uncertainity principle [26]. The Shannon entropy provides a global measure of the electron density spread, whereas the Fisher Information (FI) is defined as [27]

$$
F_{\rho}=\int \frac{|\nabla \bar{\rho}(\mathbf{r})|^{2}}{\bar{\rho}(\mathbf{r})} d \mathbf{r}
$$

for the position space and

$$
F_{\pi}=\int \frac{|\nabla \pi(\mathbf{p})|^{2}}{\pi(\mathbf{p})} d \mathbf{p}
$$

for the momentum space. Although FI quantities provide a global number per molecule, unlikely Shannon entropies, their value is largely affected by small local changes of the function as has been proved by Lopez and coworkers [28]. In this sense, the FI provides a local measure of the density sharpness. The position space FI increases when the heterogeneity of the system leads to larger 
changes of the position space density, whereas the FI in the momentum space depends on large deviations of the momentum density,

$$
\pi(\mathbf{p})=\int\left|\widetilde{\Psi}\left(\mathbf{p}_{\mathbf{1}}, \mathbf{p}_{\mathbf{2}}, \ldots, \mathbf{p}_{\mathbf{N}}\right)\right|^{2} d \mathbf{p}_{\mathbf{2}} d \mathbf{p}_{\mathbf{3}} \ldots d \mathbf{p}_{\mathbf{N}}
$$

and can be obtained from the Fourier transform that connects the momentum space wave function with the position space one

$$
\begin{aligned}
\widetilde{\Psi}\left(\mathbf{p}_{\mathbf{1}}, \mathbf{p}_{\mathbf{2}}, \ldots, \mathbf{p}_{\mathbf{N}}\right)= & (2 \pi)^{-3 N / 2} \int \Psi\left(\mathbf{r}_{1}, \mathbf{r}_{\mathbf{2}}, \ldots, \mathbf{r}_{\mathbf{N}}\right) \times \\
& \exp \left[-i\left(\mathbf{p}_{\mathbf{1}} \cdot \mathbf{r}_{\mathbf{1}}+\mathbf{p}_{\mathbf{2}} \cdot \mathbf{r}_{\mathbf{2}}+\ldots+\mathbf{p}_{\mathbf{N}} \cdot \mathbf{r}_{\mathbf{N}}\right)\right] d \mathbf{r}_{1} d \mathbf{r}_{\mathbf{2}} \ldots d \mathbf{r}_{\mathbf{N}}
\end{aligned}
$$

These information theory indicators have been used in the past to characterize the electronic structure of molecules and chemical reactions [23, 29, 30]. Some authors [29,30] have linked these concepts of information theory to particular chemical reactivity, attributing enhanced sensitivity to changes on the position and momentum densities along the chemical reaction paths [29]. In this work we will examine the description of the harpoon mechanism afforded by these indicators.

\section{Computational Details}

We study the bond dissociation mechanism of ten diatomic molecules $(\mathrm{BH}$, $\mathrm{BeH}, \mathrm{CO}, \mathrm{F}_{2}, \mathrm{LiF}, \mathrm{LiH}, \mathrm{H}_{2}, \mathrm{He}_{2}, \mathrm{~N}_{2}$ and $\mathrm{O}_{2}$ ) in their ground state and the first excited state of the same symmetry and spin multiplicity than the ground state for $\mathrm{LiH}$ and $\mathrm{LiF}$. Born-Oppeheimer, full configuration interaction (FCI) calculations of $\mathrm{H}_{2}, \mathrm{He}_{2}, \mathrm{LiH}, \mathrm{BH}$ and $\mathrm{BeH}$ have been performed with a modified version of Knowles and Handy program [31], whereas Gaussian 09 [32] was used to obtain complete active space self-consistent field (CASSCF) energies. Namely, CAS(6,6), CAS(10,6), CAS(8,6), CAS(6,6) and $\mathrm{CAS}(6,6)$ single-point evaluations were performed for $\mathrm{N}_{2}, \mathrm{~F}_{2}, \mathrm{O}_{2}, \mathrm{LiF}$ and $\mathrm{CO}$, respectively. The aug-cc-pVDZ basis was employed in all cases. The $\mathrm{LiF}$ valence CASSCF calculation presents a wave function and energy discontinuity around the avoided crossing point [33. The latter problems are avoided if FCI is used or if, more conveniently, a state-average CASSCF calculation is performed [33, 34]. The state-average CAS computation produces a qualitatively correct result with an avoided crossing at about $4 \AA$ in contrast to the FCI results that place the avoided crossing at $6-6.5 \AA$ [34]. In this paper we have performed a four-state average $\operatorname{CAS}(6,6)$ with equal weights, which gives a qualitatively correct, discontinuity-free, description of 
the process that is sufficient for the purposes of this work. A six-state average CAS $(6,6)$ calculation was also performed for CO.

The quantum-theory of atoms-in-molecules (QTAIM) 19] calculations have been performed with AIMall package [35, and the topological fuzzy Voronoi cells (TFVC) computations have been done using APOST-3D software [36]. The TFVC is a three-dimensional atomic partition based on fuzzy atoms that produces results very similar to those obtained with QTAIM at a much lower computational cost [37. The pair densities (2-PD) have been obtained with DMN 38] program and inputed into ESI-3D 39] to produce the ESI and atomic populations. The ELF analysis has been performed with a modified version of the ToPMoD program [40] using the Hartree-Fock like approximation of the 2-PD in the computation of the ELF values. This approximation is needed in order to preserve the antisymmetry property of the 2-PD that other more sophisticated approximations do not fulfill and provides a very accurate description of the ELF even for stretched bonds [41. The calculation of information theory quantities has been done with the RHO-OPS code developed in our group [42].

\section{Results}

\section{$4.1 \quad \mathrm{LiH}$}

$\mathrm{LiH}$ is the smallest ionic molecule that is formed through the harpoon mechanism. The electron affinity of hydrogen is quite different from the ionization potential of lithium and, therefore, the electron transfer in $\mathrm{LiH}$ occurs at shorter distances than in alkali halides. In this section we will analyze two ${ }^{1} \Sigma^{+}$adiabatic states of $\mathrm{LiH}$, namely, $\mathrm{X}^{1} \Sigma^{+}$and $\mathrm{A}^{1} \Sigma^{+}$. The former state dissociates into $\mathrm{H}\left({ }^{2} S\right)+\mathrm{Li}\left({ }^{2} S\right)$ and the latter into $\mathrm{H}\left({ }^{2} S\right)+\mathrm{Li}\left({ }^{2} P\right)$. These two states involve the crossing between three diabatic states: the ionic ground state and the two lowest-lying covalent states. The $\mathrm{X}^{1} \Sigma^{+}$state results from the crossing between the first ionic and covalent diabatic states, showing an avoided crossing with the $\mathrm{A}^{1} \Sigma^{+}$curve at about $3 \AA$. The $\mathrm{A}^{1} \Sigma^{+}$ state is characterized by two avoided crossings: the first one has just been described and the second avoided crossing at about $6 \AA$ results from the interaction between the ionic and the second covalent states. Therefore, the $\mathrm{X}^{1} \Sigma^{+}$ground state is characterized by an ionic bonding at the equilibrium but its covalent character increases as the interatomic distance stretches. The $\mathrm{A}^{1} \Sigma^{+}$state is even more interesting because as the bond stretches one expects two changes in its character: from rather covalent to ionic and back to covalent. The presence of two crossings furnishes the $\mathrm{A}^{1} \Sigma^{+}$curve with a characteristic flat region that leads to huge anharmonic effects and anomalous 
spectroscopic constants [43. LiH lowest-lying states have been widely studied in the past [5, 44, 45 due to the confluence of these peculiar features, which make them a formidable playground to test modern electronic structure methods. The ground state electron harpooning process has been studied by means of chemical bonding descriptors [2, 3, 11] but, to our knowledge, the excited state has never been explored from the chemical bonding viewpoint.

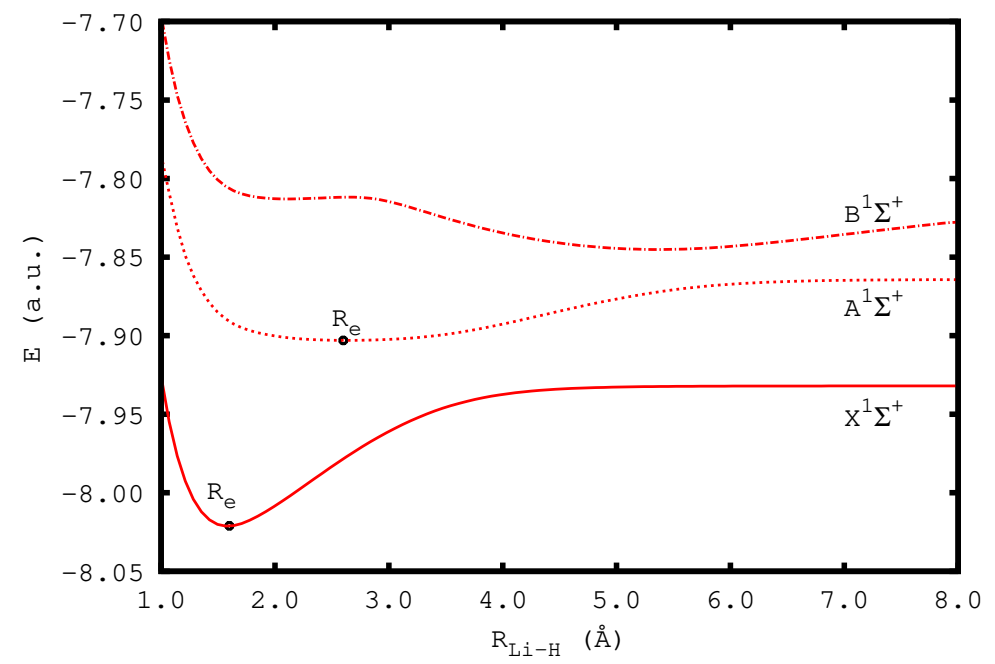

Figure 1. Potential Energy Curve (PEC) of the $\mathrm{LiH}$ for the ground state $\left(\mathrm{X}^{1} \Sigma^{+}\right)$and the two lowest-lying ${ }^{1} \Sigma^{+}$excited states $\left(\mathrm{A}^{1} \Sigma^{+}\right.$and $\left.\mathrm{B}^{1} \Sigma^{+}\right)$. Energy in a.u.

Let us first describe the electron exchange between both atoms that occur upon stretching the $\mathrm{LiH}$ bond in the two electronic states. To this aim, the most straightforward analysis consists in checking the atomic populations along the energy curves. Fig. 2 contains the change in the QTAIM population of the most electropositive atom as the molecule dissociates into two atomic fragments (only molecules that present electron reorganization have been included). The total density transferred from hydrogen to lithium is about 0.9 electrons in the ground state, where the molecule passes through an avoided crossing that changes its bonding character from ionic to covalent. Conversely, the overall transfer in the $\mathrm{A}^{1} \Sigma^{+}$state is barely 0.2 electrons. As the bond stretches the electron passes from lithium to hydrogen until about 0.5 electrons are completely transferred. This process takes places as the curve goes through the first avoided crossing and the bond becomes more ionic. At about $2 \AA$ stretching, the electron transfer is reversed and 
the lithium withdraws electron charge when passing by the second avoided crossing. About 0.7 electrons are transferred in this second step and the bond evolves from ionic to rather covalent. The covalent character of the molecule in the $\mathrm{A}^{1} \Sigma^{+}$state at geometries near the equilibrium is not as strong as one would expect in ground-state covalent molecules, however, the obtained ESI values are larger than in pure ionic molecules and unprecedented of excited states stretched bonds (vide infra).

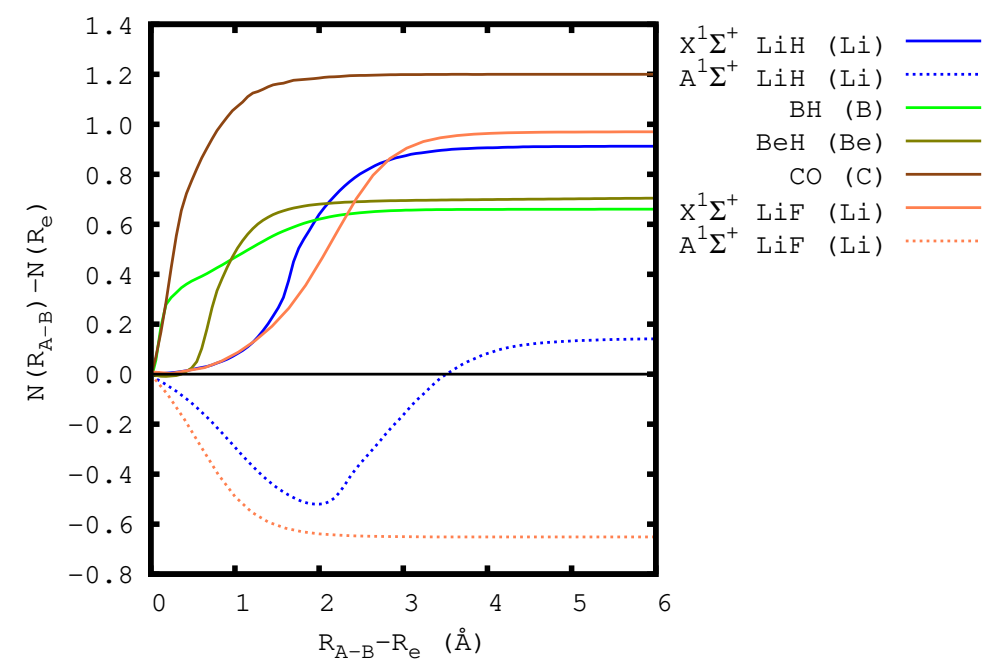

Figure 2. Change in the atomic QTAIM population of the most electropositive atom along the bond stretching in the series of studied molecules that present electron reorganization. Population units are electrons.

The plot of the dipole moment along the internuclear axis at different interatomic separations (Fig. 3) can be also used to monitor the electron-transfer process. For the ground-state at equilibrium $\mu=2.33$ a.u. and this value increases as the bond stretches, peaking at $2.8 A$ and then decreasing to zero as the atoms tend to isolated neutral entities. Conversely, the $\mathrm{A}^{1} \Sigma^{+}$state shows a lower dipole moment at the equilibrium with opposite sign $(\mu=-1.1$ a.u.), an indication of the more covalent nature of this bond as compared to the $\mathrm{X}^{1} \Sigma^{+}$state. The dissociation of the $\mathrm{A}^{1} \Sigma^{+}$state of $\mathrm{LiH}$ involves the sign change of the dipole moment as it passes through the first avoided crossing; reaches a maximum value around $5 \AA$ and then decreases to zero as it goes through the second avoided crossing.

In Fig. 4 we find the ESIs along the dissociation curves for a series of 


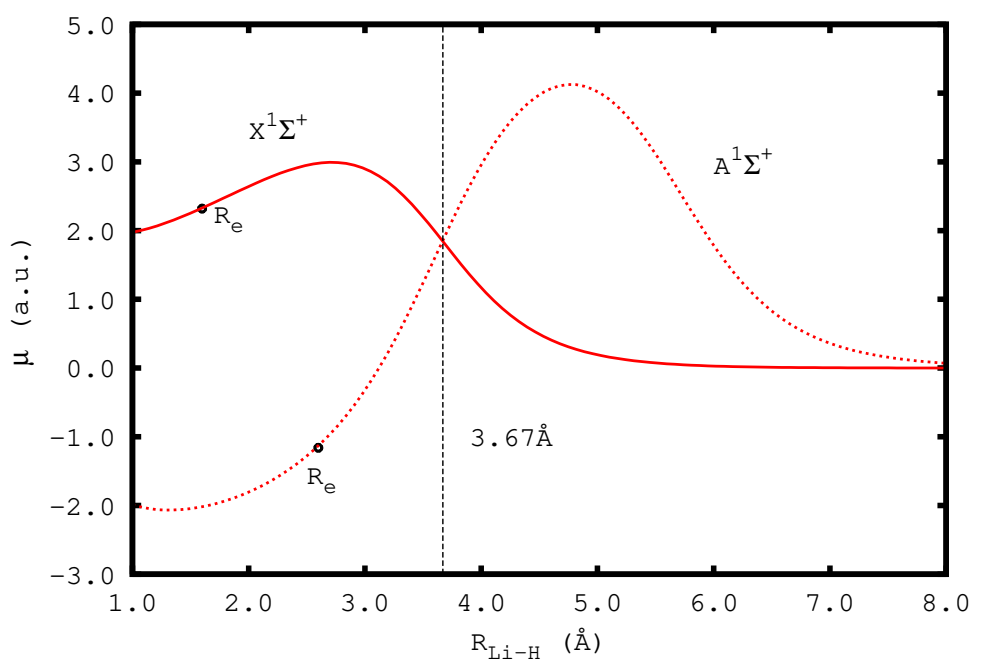

Figure 3. Dipole moment (in a.u.) along the internuclear axis for the $\mathrm{X}^{1} \Sigma^{+}$and the $\mathrm{A}^{1} \Sigma^{+}$states of $\mathrm{LiH}$ as function of the interatomic distance.

diatomic molecules. The ground-state LiH ESI has a low value (0.19) at the equilibrium that slightly decreases as the bond is stretched but soon enough increases as the covalent contribution plays a more important role in the description of the $\mathrm{X}^{1} \Sigma^{+}$state, reaching a maximum around $3.3 \AA$ and dying off at large interatomic separations. The ESI maximum is the point with the largest electron sharing between the atoms, i.e., the point at which the electron is least attached to either fragment (although, not loose enough to form a separate entity). Indeed, the ESI peaks at the maximal transfer variation position, i.e., the position at which the variation of electron population with the interatomic distance is largest (see vertical lines in the inset plot of Fig. (4). Furthermore, at this point 0.5 electrons have been transferred from $\mathrm{Li}$ to $\mathrm{H}$ when we compare their atomic populations with the ones of the isolated neutral atoms as already reported by Pendás and coworkers [10]. The ESI can be thus used to locate the position at which the electron remains in no one's land, which one identifies with the avoided crossing region. The analysis of the $\mathrm{A}^{1} \Sigma^{+}$state reveals a similar ESI profile for interatomic distances from $1 \AA$ to $3 \AA$ than for $\mathrm{X}^{1} \Sigma^{+}$state. Despite the ESI values in this range are similar, the nature of the bond is completely different. Let us analyze $R=1.6 \AA$, which is the equilibrium geometry for the $\mathrm{X}^{1} \Sigma^{+}$state. At this distance the ground state is mostly ionic (low ESI, atomic charges close to +1 and -1 and the dipole moment vector pointing towards hydrogen), whereas the $\mathrm{A}^{1} \Sigma^{+}$state is covalent antibonding because 
the charges are virtually zero for each atom and the dipole moment vector is of the same magnitude but opposite sign. The LiH ESI value at equilibrium for the $\mathrm{A}^{1} \Sigma^{+}$state $\left(R_{e}^{\mathrm{A}^{1} \Sigma^{+}}=2.6 \AA\right)$ is larger because the molecule becomes more covalent as it approaches the equilibrium distance in line with the more covalent character one expects from the excited state $\left(\delta^{\mathrm{X}^{1} \Sigma^{+}}(\mathrm{Li}, \mathrm{H})=0.19\right.$ vs. $\left.\delta^{\mathrm{A}^{1} \Sigma^{+}}(\mathrm{Li}, \mathrm{H})=0.30\right)$. For bond lengths beyond $3 \AA$ the ESI curves of the two states deviate from each other and the $\mathrm{A}^{1} \Sigma^{+}$state presents rather constant values (ca. 0.5) up to $5 \AA$; from this point the ESI decreases until it reaches zero beyond $8 \AA$. Interestingly, the ESI of the $\mathrm{A}^{1} \Sigma^{+}$state attains a second maximum around the position of the second avoided crossing. In the formation of $\mathrm{LiH}$ in the $\mathrm{A}^{1} \Sigma^{+}$state the two ESI maxima correspond to points at which there is a maximal transfer variation; around the first maximum, lithium passes electron density to hydrogen and the opposite occurs in the vicinity of the second one. The latter is actually the point in the molecule formation at which 0.5 electrons have been transferred from Li to $\mathrm{H}$.

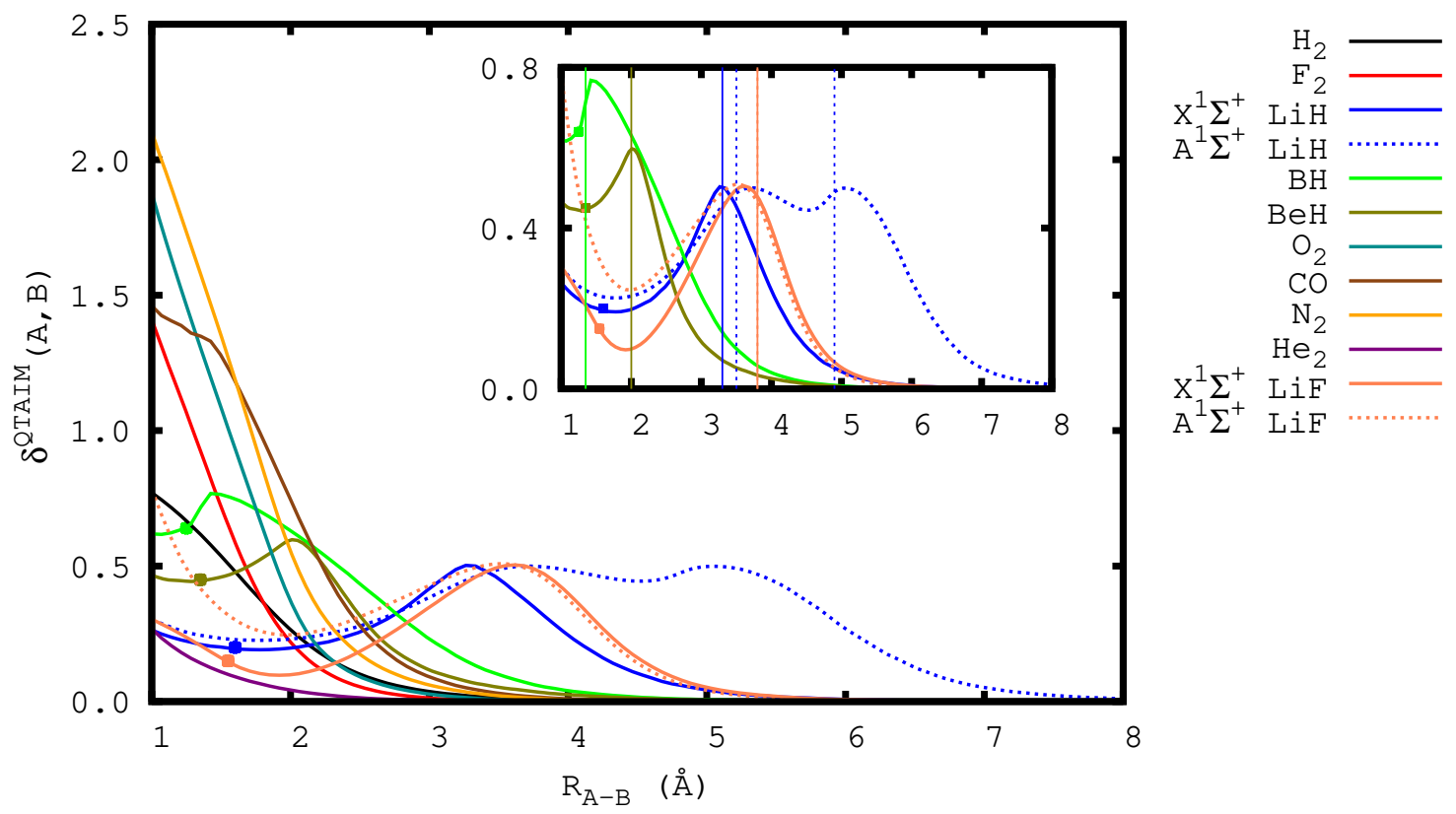

Figure 4. Electron sharing indices calculated using QTAIM partition for the series of diatomic molecules. Solid and dotted lines are used for ground states and excited states, respectively. The solid points indicate the position of the equilibrium distance and the vertical lines in the inset plot mark the position of the maximal electrontransfer variation points (see text). ESI units are electron pairs. 
At this point, one should ask whether other tools to characterize the electronic structure of molecules are capable of following the electron as it jumps from one atom to another. The ELF has been successfully used in the characterization of localized electrons as those occuring in covalent bonds, lone pairs [46] or even isolated electrons in ionic compounds [47] and it has been also used to analyze some electron-transfer processes [8,9]. However, the investigation of the ELF for $\mathrm{LiH}$ dissociation [9] does not reveal a separate basin that can be identified with the electron transferred. The process is characterized by a progressive elongation of hydrogen's valence basin that eventually splits and is absorbed by lithium's valence basin but, at no point, it becomes a separate, distinctive basin.

QTAIM analysis along the two electronic states puts forward that there is no non-nuclear attractor of the density and no Laplacian basin in the bonding region corresponding to the transferred electron. Indeed, depending on the interatomic separation, the electron is located in the lithium or the hydrogen atom but it is never found in a separate entity. Therefore, a three-dimensional representation of these functions cannot be used to pinpoint the electron position as it jumps between atoms.

The electron jump process is difficult to identify by the isosurface representation but it is easily monitored by ELF profiles along the internuclear axis. Fig. 5 includes several profiles of the ELF along the molecular axis of LiH's $\mathrm{X}^{1} \Sigma^{+}$state at different interatomic distances. A vertical dashed line marks the minimal density position between $\mathrm{Li}$ and $\mathrm{H}$ in the molecular axis. The latter is known in the QTAIM framework as the bond critical point $(\mathrm{BCP})$, and in these plots the BCP indicates the atomic boundary along the internuclear axis. These plots show that at about the avoided crossing region the valence basin of lithium emerges at one end of the molecule, whereas the hydrogen valence basin starts to split into two but it still belongs to the $\mathrm{H}$ atomic QTAIM basin. At $3.3 \AA$ the hydrogen peak splits into two and the second peak carrying the transferred electron crosses the boundary between QTAIM atomic basins afterwards. At $5 \AA$ the process is almost complete and the lithium valence basin is fully characterized (see the two peaks around the lithium atom in the one-dimensional plot of Fig. 5). At no point during the dissociation the (one-dimensional) maximum between $\mathrm{Li}$ and $\mathrm{H}$ in Fig. 5 becomes a three-dimensional ELF maximum that would give rise to a separate basin. In this sense, a one-dimensional ELF representation is more useful than the usual isosurface picture in order to trace the electron motion in the reaction mechanism. Inspection of the $\mathrm{A}^{1} \Sigma^{+}$state ELF profile along the molecular axis reveals the peak formation after the first avoided crossing and the peak splits and moves towards lithium until it has completely passed the 
atomic QTAIM boundary after the second avoided crossing (see Fig. 6).
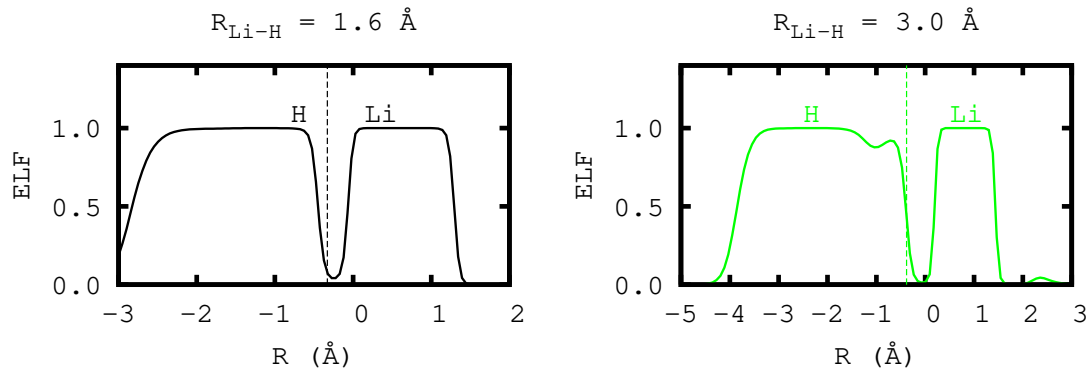

$\mathrm{R}_{\mathrm{Li}-\mathrm{H}}=3.7 \AA$
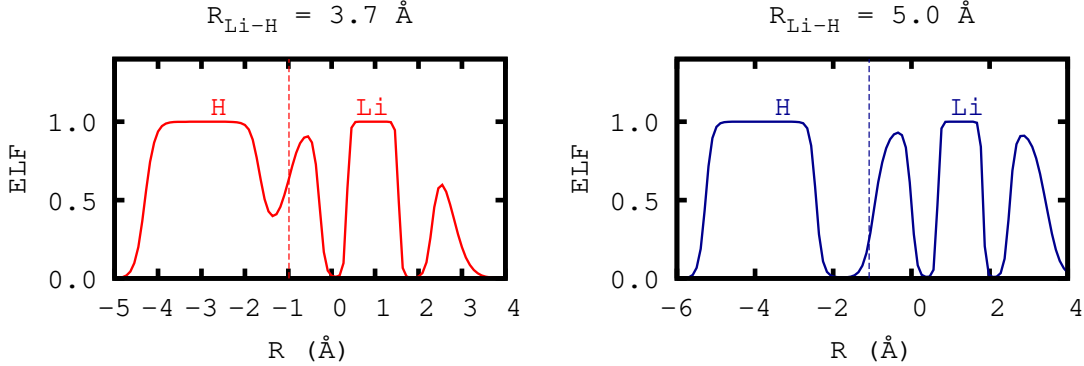

Figure 5. The ELF profile of the $\mathrm{X}^{1} \Sigma^{+}$state of LiH along the internuclear axis for several values of the interatomic distance. The zero is located at the center of mass and the vertical dashed line indicates the bond critical point. The first avoided crossing occurs at about $3 \mathrm{~A}^{\circ}$.

Similarly, the molecular-axis scan of the Laplacian of the electron density at different interatomic separations reveals the harpooning mechanism. In Fig. 7 we represent the negative values of the Laplacian of the electron density along the molecular axis at different interatomic distances; we have used logarithmic scale to allow an easy identification of the peaks and dashed vertical lines to indicate the BCPs. For the $\mathrm{X}^{1} \Sigma^{+}$state at $2 \AA$, the peak corresponding to the hydrogen atom starts to expand, splitting into two separate peaks after $2.5 \mathrm{~A} 11$ Then, the two peaks separate from each other and, eventually, the new peak separates completely from hydrogen peak and becomes the valence basin of lithium. Interestingly, the new peak containing the transferred electron starts crossing the QTAIM atomic boundary at the first avoided crossing. For the $\mathrm{A}^{1} \Sigma^{+}$state, the peak between $\mathrm{Li}$ and $\mathrm{H}$ shows at about $3.7 \AA$. As the

${ }^{1}$ Setting the position of the peaks' separation is not exempt of numerical error. We have set the peaks' separation after $2.5 \AA$ by neglecting values of the Laplacian below $10^{-4}$. 

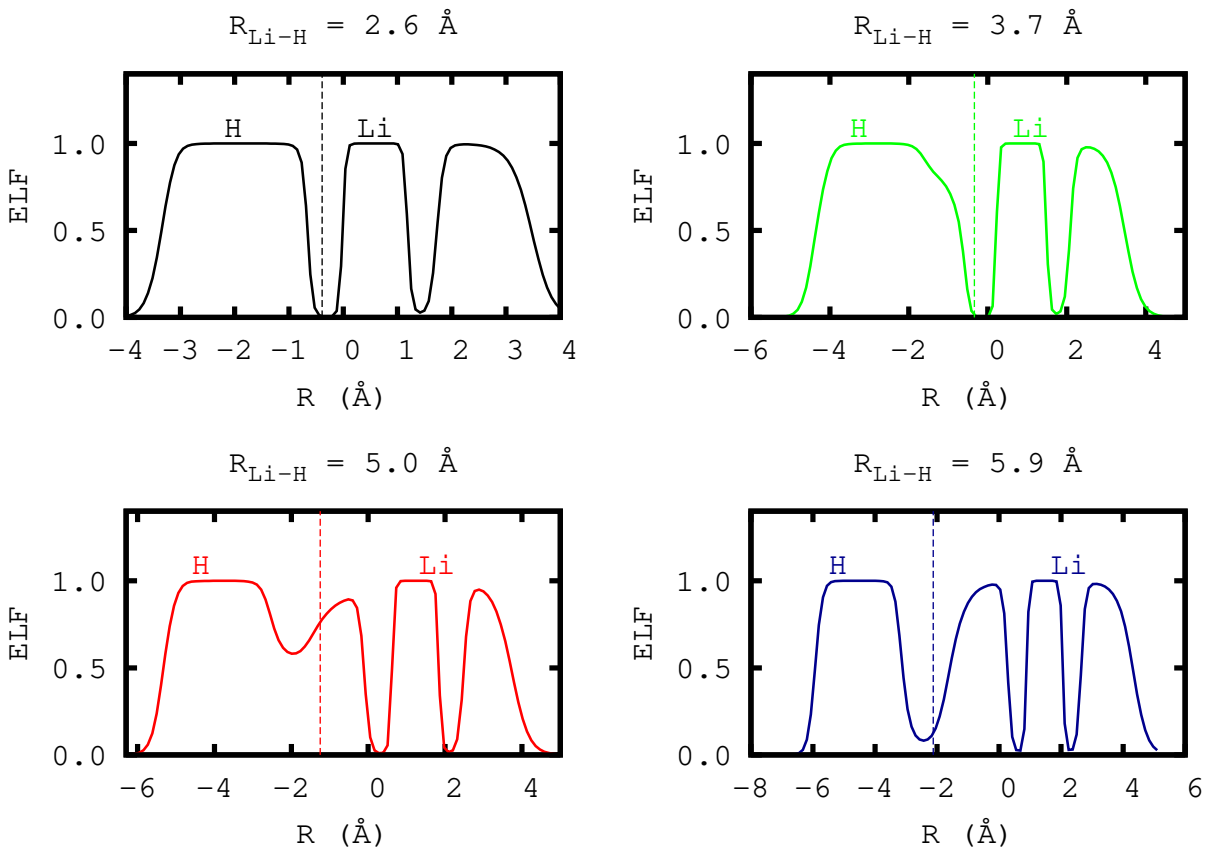

Figure 6. The ELF profile of the $\mathrm{A}^{1} \Sigma^{+}$state of $\mathrm{LiH}$ along the internuclear axis for several values of the interatomic distance. The zero is located at the center of mass and the vertical dashed line indicates the bond critical point. The first avoided crossing occurs at about $3 \AA$ and the second at $6 \AA$.

distance elongates, the peak broadens and it begins to cross the QTAIM atomic boundary after $4.0 \AA$ and it is completely transferred when the molecule passes the second avoided crossing (see Fig. 7 bottom picture). Therefore, according to the ELF-QTAIM description the electron transfer of the $\mathrm{A}^{1} \Sigma^{+}$state also takes place between the two avoided crossings.

\subsection{Other Bond Formations}

In this section we analyze the formation process of other diatomic molecules in their ground state from the isolated neutral atoms. First, we have chosen two additional molecules, $\mathrm{BeH}$ and $\mathrm{LiF}$, which are also formed through an electron harpooning mechanism due to ionic and covalent diabatic state crossings. The former consists of $\mathrm{Be}$, which has an ionization potential larger than $\mathrm{Li}$ and, therefore, contributes to a larger energy difference with hydrogen's electron affinity than $\mathrm{LiH}$. As a result, the electron jump takes places at quite short distances. Conversely, $\mathrm{F}$ has a larger electron affinity than $\mathrm{H}$, giving a small IP-EA value for the atoms in $\mathrm{LiF}$. The electron jump in LiF thus occurs at 


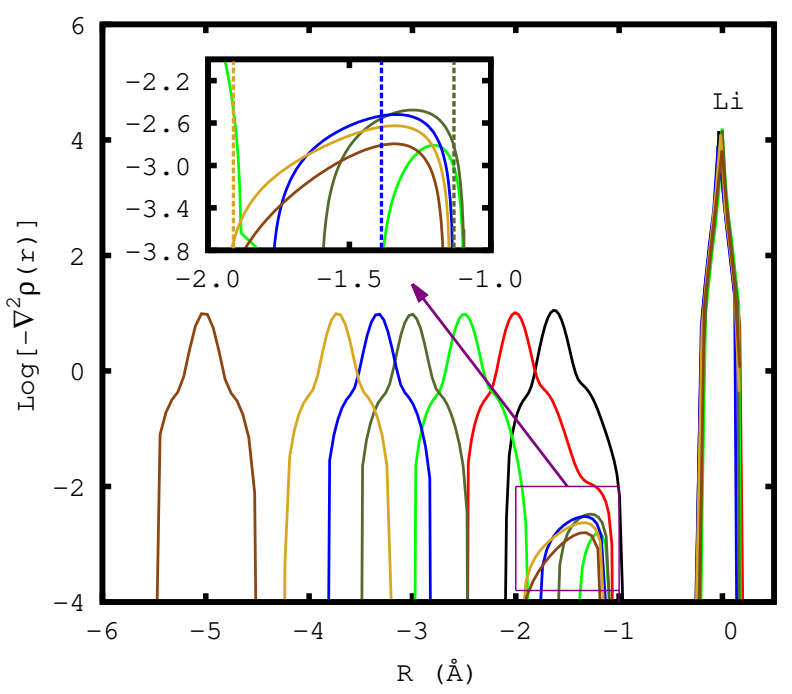

$1.6 \AA-$
$2.0 \AA-$
$2.5 \AA-$
$3.0 \AA-$
$3.3 \AA-$
$3.7 \AA-$
$5.0 \AA-$

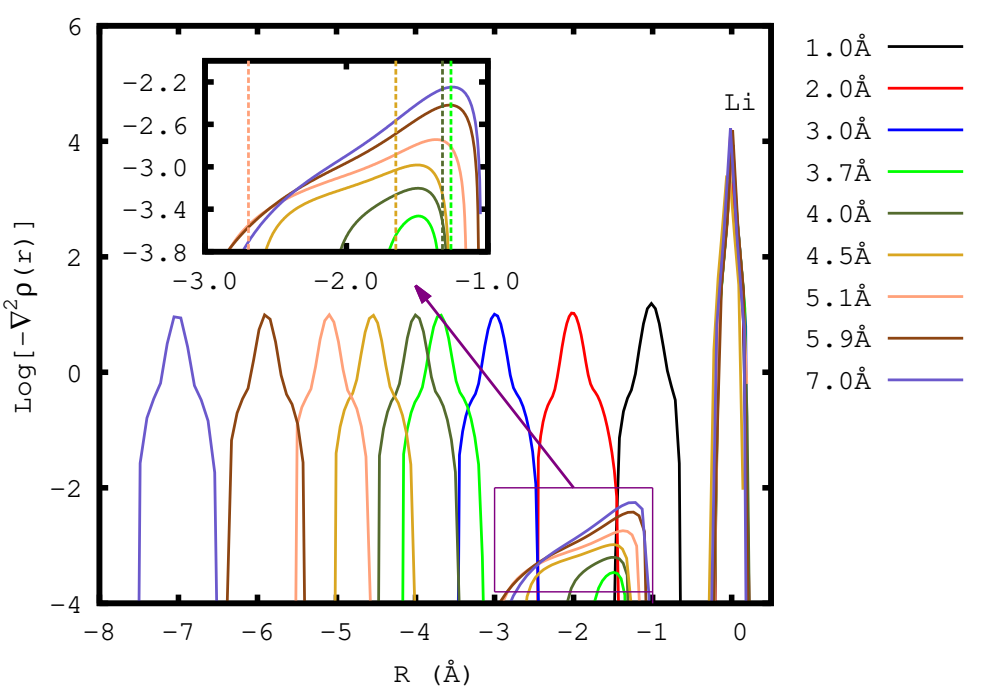

Figure 7. The negative values of the Laplacian of the electron density of $\mathrm{LiH}$ along the internuclear axis for several interatomic distances (top $\mathrm{X}^{1} \Sigma^{+}$, bottom $\mathrm{A}^{1} \Sigma^{+}$). All molecules locate $\mathrm{Li}$ atom in the z-axis at zero for comparison. The vertical dashed lines in the inset plot indicate the bond critical points. Laplacian units are a.u.

large interatomic separations but the state-average CASSCF description of the process underestimates the distance at which the avoided crossing occurs (vide supra). Notwithstanding, it does not affect the qualitative bonding picture of $\mathrm{LiF}$ or the bond mutation process around the avoided crossing. Second, we analyze $\mathrm{H}_{2}, \mathrm{O}_{2}, \mathrm{~N}_{2}, \mathrm{~F}_{2}, \mathrm{He}_{2}, \mathrm{BH}$ and $\mathrm{CO}$, which are mostly 
included for comparison. These molecules present different bonding patterns from weakly bonded to covalent single-, double-, and triple-bonds, as well as a polar covalent bond. An example of the latter is the $\mathrm{CO}$ molecule and its formation also implies an important electron reorganization process in spite of the fact that the mechanism does not involve electron harpooning.

Let us first analyze $\mathrm{BeH}$ and LiF. Fig 8 shows the two lowest-lying ${ }^{1} \Sigma^{+}$ states of $\mathrm{LiF}$ : the $\mathrm{X}^{1} \Sigma^{+}$and $\mathrm{A}^{1} \Sigma^{+}$states. The dipole moment of both states of $\mathrm{LiF}$ coincides at a distance close to the avoided crossing (ca. $3.6 \AA$ ) (see Fig S1 in the Supp. Info). The ESI profile in Fig 4 also shows a maximum around the avoided-crossing interatomic distance for both states. The ESI profile of LiF's $\mathrm{A}^{1} \Sigma^{+}$state shows larger (slightly more covalent) values than the $\mathrm{X}^{1} \Sigma^{+}$state before the avoided crossing but the rest of the ESI profile is very similar. The avoided crossing point is not only the geometry at which the ESI is maximum, but also the point at around which there is a maximal transfer variation and a net transfer of 0.5 electrons from Li to $\mathrm{F}$ as compared to its isolated neutral counterparts. The ELF and the Laplacian profiles along the internuclear axis show a separate peak (corresponding to the transferred electron from $\mathrm{F}$ to $\mathrm{Li}$ ) that emerges before the avoided crossing and it crosses the QTAIM atomic boundary around the avoided crossing position (see Figs. S2 and S3).

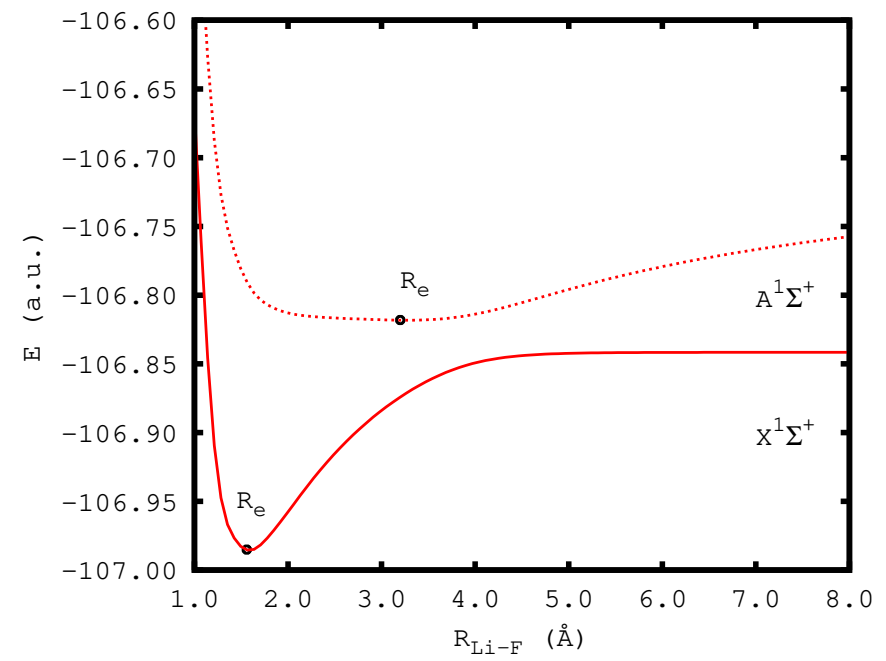

Figure 8. Potential Energy Curve (PEC) of the LiF for the ground state $\left(\mathrm{X}^{1} \Sigma^{+}\right)$and the lowest-lying $\mathrm{A}^{1} \Sigma^{+}$excited state. Energy in a.u. 
Unlike $\mathrm{LiH}$ and $\mathrm{LiF}, \mathrm{BeH}$ 's $\mathrm{X}^{2} \Sigma^{+}$ground state avoided crossing occurs at very short distances (ca. $2.2 \AA$ [48]). The ESI profile reveals a maximum at this position that is also the same position at which the transfer variation is maximal and 0.5 electrons have been transferred from $\mathrm{H}$ to Be. The ELF and Laplacian show features similar to those of $\mathrm{LiH}$ and $\mathrm{LiF}$, with the peak corresponding to the electron carried from one atom to another crossing the QTAIM atomic boundary around the avoided crossing (see Figs S4 and S5).

$\mathrm{BH}$ in its $\mathrm{X}^{1} \Sigma+$ state presents a rather large ESI value for an ionic species $(\delta(B, H)=0.6)$ and, actually, the valence bond description assigns a covalent character to this bond 49 . The ground state energy curve does not present avoided crossings that rapidly could change the bond character from ionic to covalent or viceversa. Therefore, all evidences suggest a electron reorganization without harpoon mechanism. Notwithstanding, our results show otherwise. The ESI profile is characteristic of a harpooning mechanism taking place at very short distances (ca. 1.5A). A fact that is confirmed by ELF and Laplacian profiles along the molecular axis (see Figs S6 and S7), which show that ELF and Laplacian hydrogen's peak splits into two and passes the atomic QTAIM boundary from $\mathrm{H}$ to B at the vicinity of the ESI maximum, where the molecule experiences its maximal transfer variation and 0.5 electrons have already passed from $\mathrm{H}$ to $\mathrm{B}$. Therefore, one is deemed to conclude that the formation of $\mathrm{BH}$ also takes places through a harpoon mechanism without the existence of an avoided crossing.

According to QTAIM population analysis (see Fig. 2), carbon withdraws more than one electron from oxygen in the $\mathrm{CO}$ molecule. Unlike the $\mathrm{LiH}$ or LiF, the electron-density transfer does not imply a large change in the dipole moment. Although the shape of the dipole moment profile along the dissociation is similar in all electron-transfer reactions, the reaction in $\mathrm{CO}$ (see Fig. S8) takes places through very small values of the dipole moment (max. 0.3, min. -0.1). The ESI profile exhibits a smoothly decreasing profile that dies off after $4 \AA$. There is, therefore, no ESI maximum at stretched distances but the maximal transfer variation coincides again with the point where 0.5 electrons have been transferred from $\mathrm{C}$ to $\mathrm{O}$. The Laplacian and the ELF scans along the interatomic axis describe the dissociation process as the splitting of the bonding peak (corresponding to the triple bond) into two peaks (see Fig. S9 and S10 in Supp. Info.).

The rest of the molecules are calculated to afford a comparison between the above-described electron reorganization processes and other reactions. The ESI profiles of $\mathrm{F}_{2}, \mathrm{O}_{2}$ and $\mathrm{N}_{2}$ exhibit a sigmoidal shape that Pendás and coworkers identify with the breaking of covalent bonds, whereas the $\mathrm{He}_{2}$ ESI dissociation 
curve decays exponentially, in line with noncovalent interactions [10]. The ELF and Laplacian scan along the molecular axis do not have much interest because they do not reveal additional features as compared to the isosurface representations discussed elsewhere [9].

\subsection{Information theory indicators}

Finally, let us examine the profiles provided by five indicators defined in the framework of information theory. Fig. 9 collects FI and Shannon indicators (see. Eqns. 6.9) at the dissociation limit minus its value at different interatomic separations $\left(\Delta S_{\rho}, \Delta S_{\pi}, \Delta F_{\rho}, \Delta F_{\pi}\right.$ and $\left.\Delta S_{T}\right) 1$ Since the molecules analyzed undergo different bond formation mechanisms one would expect some distinguishing features in the representation of these indicators along the dissociation curves.

$\Delta S_{\rho}$ shows that molecules that undergo an electron-transfer process show larger differences with respect to its value at infinite separation. These molecules experience an internal electron reorganization at stretched distances that is expected to affect its $S_{\rho}$. In this regard, $\mathrm{LiF}$ and $\mathrm{LiH}$ experience a long-range electron exchange that explains why these molecules converge to $\Delta S_{\rho} \rightarrow 0$ at larger distances than the other molecules. On the other hand, it is not clear why the $\mathrm{H}_{2}$ profile lies very close to $\mathrm{BeH}$ and $\mathrm{BH}$ curves or why covalent molecules show $\Delta S_{\rho}$ profiles very similar to $\mathrm{He}_{2}$. Therefore, it does not seem to be an obvious connection between $\Delta S_{\rho}$ profiles and the bond formation mechanim in diatomics. Similar conclusions can be drawn from the inspection of $\Delta S_{T}$ profiles.

$\Delta F_{\rho}$ shows maximal values more or less close to the avoided crossings for $\mathrm{LiH}, \mathrm{LiF}$ and $\mathrm{BeH}$, whereas $\Delta F_{\rho}$ decays exponentially for the other molecules except $\mathrm{He}_{2}$, which shows a somewhat sigmoidal shape. In this sense, the profile of $\Delta F_{\rho}$ could provide a distinctive shape for each type of mechanism, excluding $\mathrm{H}_{2}$ whose shape does not match the other covalent molecules. On the other hand, there does not seem to be a way to distinguish between single-, double- or triple-bond dissociations.

As far as the magnitudes based on the momentum density $\left(\Delta S_{\pi}\right.$ and $\left.\Delta F_{\pi}\right)$ go, we can see that the molecules involving a electron-transfer harpoon mechanism present larger differences with respect to the value at infinite separation. One more time, hydrogen molecule is an exception to this rule.

\footnotetext{
${ }^{1}$ Please notice that these quantities are not size extensive and, therefore, their values can not be compared with the sum of the isolated fragments.
} 

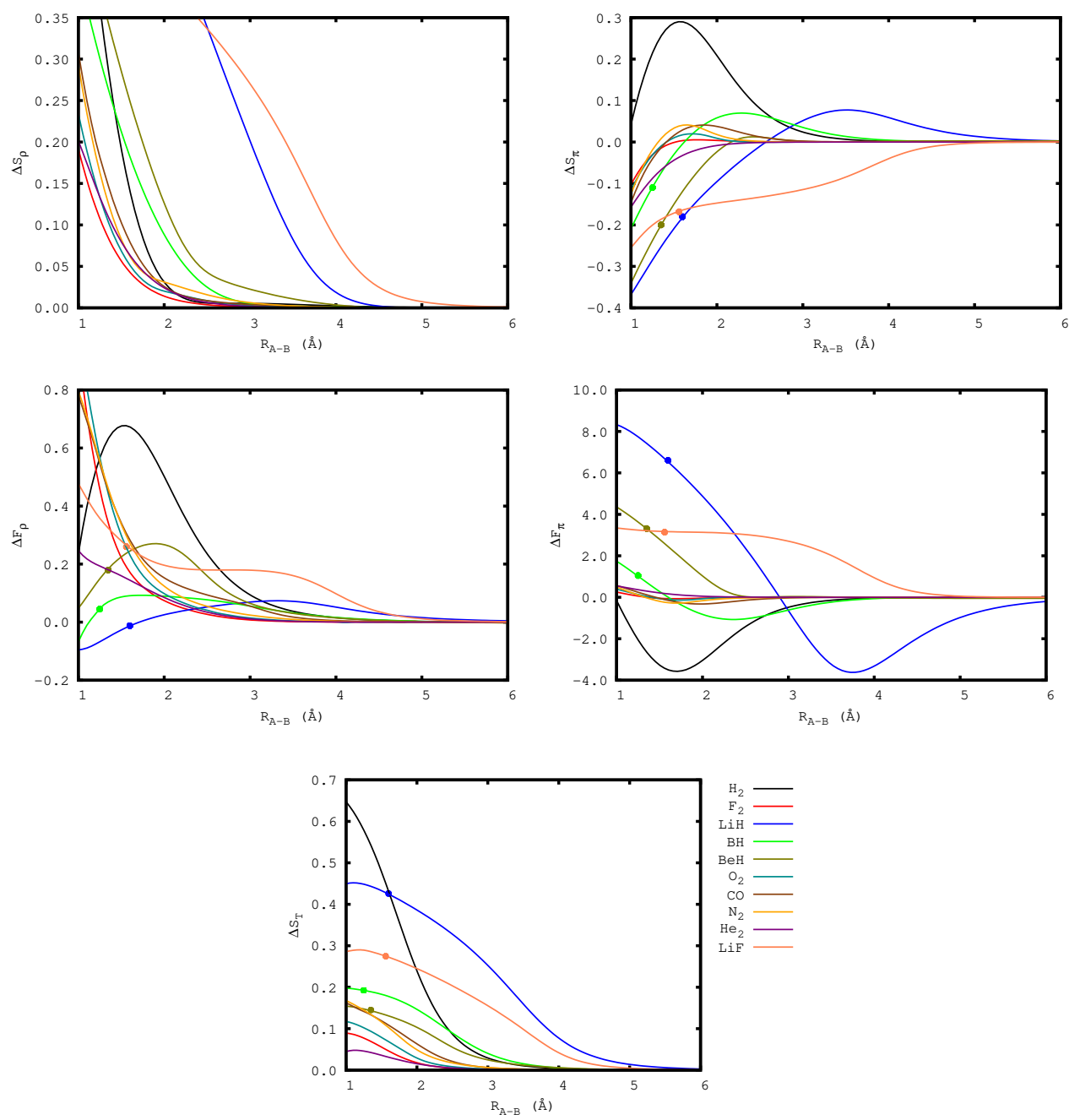

Figure 9. From left to right and top to bottom: (a) Shannon Entropy in the position space (see Eq.6); (b) Shannon Entropy in the momentum space (see Eq. 77); (c) Fisher Information in the position space (see Eq. 8); (d) Fisher Information in the momentum space (see Eq. 9); (e) Total Shannon Entropy $\left(S_{T}=S_{\rho}+S_{\pi}\right.$ ); plots represent the values at the dissociation limit minus the value at different interatomic separations for the series of diatomic molecules taken into study. Information theory descriptors units are a.u.

\section{Discussion and Conclusions}

The lowest-lying states of $\mathrm{LiH}$ have been widely used to develop and calibrate many different methods in quantum mechanics. In this paper 
we have shown that the electron-transfer processes occurring in these two states are a difficult test for chemical bonding descriptors and can be used with a two-fold purpose: (i) to test new bonding descriptors on its ability to recognize the harpoon mechanism and (ii) to evaluate the goodness of new atomic partitions to reproduce the maximal ESI value in several reactions.

Despite the harpoon mechanism involves the electron transfer at large interatomic distances, this reaction can be mistaken by a regular electron reorganization process that does not involve a harpooning process. Indeed, in Fig. 2 one finds the population analysis of $\mathrm{BeH}$, which is formed by the harpoon mechanism at short interatomic distances. The Be population profile could be easily confused with a regular electron reorganization process as that taking place in $\mathrm{CO}$. Therefore, the atomic population can be used to monitor the electron-density exchange between atoms but it cannot be always used to discriminate the reaction mechanism. In all studied mechanisms, the maximal transfer variation point along the bond formation occurs when about half electron has been transferred from one atom to another. If the process takes places through a harpoon mechanism, this point of the reaction path coincides with the avoided crossing (if there is any). Thus far, the only bonding descriptor that had actually been shown to identify the harpoon mechanim from other processes is the ESI [2, 3], which shows a maximum around the avoided crossing (see Fig. (4). The existence of a maximum of the ESI does not guarantee the existence of an avoided crossing as proved by the $\mathrm{BH}$ molecule. However, the ESI provides a simple tool to identify the existence of the avoided crossing if there is such point in the potential energy curve.

Some of us have shown in the past that atomic populations and multicenter ESI are severely affected by the atomic partition [50, but, in the big picture, atomic partitions do not have a large effect on the relative two-center ESI values [51, 1 However, Fig 4 shows that the presence of a maximum ESI value in bonds formed by the harpoon mechanism is a very constrictive test for atomic partitions. The TFVC is among the few atomic partitions that can pass such test (see Fig. S11), even though the ESI values calculated with this partition fail to identify the bond in $\mathrm{LiH}$ 's $\mathrm{A}^{1} \Sigma^{+}$state as more covalent than the bond in the $\mathrm{X}^{1} \Sigma^{+}$state at short distances $(\mathrm{R}=1-3 \AA)$.

This work also shows that ELF and Laplacian plotted along the molecular axis at different interatomic distances permit to monitor the bond formation

\footnotetext{
${ }^{1}$ The only exception are Hilbert space partitions (such as Mulliken's or Löwdin's), which show spurious results if the calculations involve a many-body wave function and its exact pair density function 52 .
} 
process better than its three-dimensional counterparts. Both tools describe the transferred electron as a one-dimensional peak that moves from one atom to another as the process takes places. The position of the peak with respect to the QTAIM boundary permits to follow this motion and shows that the peak passes from one atom to the other at the vicinity of the avoided crossing, where the ESI value and the transfer variation are maximal.

Global information theory measures based on the electron density seem to provide some distinctive features for different mechanisms of bond formation but they do not provide a convincing description that allows a clear-clut separation between the different processes. Sometimes the descriptors provide alike profiles for different bonding mechanisms and other times they provide very disparate results for similar processes. In addition, the results obtained for the $\mathrm{H}_{2}$ dissociation are not trivial to interpret and suggest that previous successful results describing chemical reaction paths were, to some extent, fortuitous [29, 30]. The information theory descriptors analyzed in the atomic regions within the molecule [21,23. or the orbital entaglement measures [53. (which can be used to locate avoided crossings) could actually provide some insights into the bonding mechanism of molecules but such study is beyond the scope of this work.

\section{Acknowledgements}

The authors thanks Prof. Bernard Silvi for providing Ref. [8].

\section{Funding}

This research has been funded by the MINECO projects CTQ2014-52525-P and CTQ2014-54306-P, the Basque Country Consolidated Group Project No. IT588-13, the FEDER grant UNGI10-4E-801 (European Fund for Regional Development), the Generalitat de Catalunya (project number 2014SGR931, Xarxa de Referència en Química Teòrica i Computacional, and ICREA 2014 prize to M.S.) and the predoctoral fellowship FPU13/00176. Excellent service by the Centre de Serveis Científics i Acadèmics de Catalunya (CESCA) and technical and human support provided by SGI/IZO-SGIker UPV/EHU are gratefully acknowledged.

\section{References}

[1] J. C. Polanyi and A. H. Zewail, Acc. Chem. Res. 28, 119 (1995). 
[2] R. Ponec and D. L. Cooper, J. Mol. Struct. (Theochem) 727, 133 (2005).

$3]$ E. Matito, M. Solà, P. Salvador, and M. Duran, Faraday Discuss. 135, 325 (2007).

[4] E. Ramos-Cordoba, E. Matito, and P. Salvador, unpublished results .

[5] B. Weiner and Y. Ohrn, J. Phys. Chem. 91, 563 (1987).

[6] P. A. Hyams, J. Gerratt, D. L. Cooper, and M. Raimondi, J. Chem. Phys. 100, 4417 (1994).

[7] J. Hernández-Trujillo and R. F. W. Bader, J. Phys. Chem. A 104, 1779 (2000).

[8] X. Krokidis, B. Silvi, C. Dezarnaud-Dandine, and A. Sevin, New J. Chem. 22, 1341 (1998).

[9] B. Silvi, I. Fourré, and E. Alikhani, Monatshefte für Chemie 136, 855 (2005).

[10] M. García-Revilla, P. L. A. Popelier, E. Francisco, and A. Martín Pendás, J. Chem. Theory Comput. 7, 1704 (2011).

[11] A. Martín Pendás, E. Francisco, and M. A. Blanco, Faraday Discuss. 135, 423 (2007).

[12] R. F. W. Bader and M. E. Stephens, J. Am. Chem. Soc. 97, 7391 (1975).

[13] X. Fradera, M. A. Austen, and R. F. W. Bader, J. Phys. Chem. A 103, 304 (1998)

[14] C. A. Coulson, Proc. Roy. Soc. A 169, 413 (1939).

[15] A. D. Becke and K. E. Edgecombe, J. Chem. Phys. 92, 5397 (1990).

[16] B. Silvi, J. Phys. Chem. A 107, 3081 (2003).

[17] E. Matito, B. Silvi, M. Duran, and M. Solà, J. Chem. Phys. 125, 024301 (2006).

[18] A. Savin, A. D. Becke, J. Flad, R. Nesper, H. Preuss, and H. G. von Schnering, Angew. Chem. Int. Ed. Engl. 30, 409 (1991).

[19] R. F. W. Bader, Atoms in Molecules: A Quantum Theory, Oxford Univ. Press, Oxford, 1990.

[20] E. Matito and M. Solà, Coord. Chem. Rev. 253, 647 (2009).

[21] R. F. Nalewajski, J. Phys. Chem. A 104, 11940 (2000).

[22] R. F. Nalewajski, Chem. Phys. Lett. 372, 28 (2003).

[23] P. Geerlings and A. Borgoo, Phys. Chem. Chem. Phys. 13, 911 (2011).

[24] C. E. Shannon and W. Weaver, The Mathematical Theory of Communication, The University of Illinois Press, Urbana, 1949.

[25] E. T. Jaynes, Phys. Rev. 106, 620 (1957).

[26] I. Bialynicki-Birula and J. Mycielsky, Commun. Math. Phys. 44, 129 (1975).

[27] R. A. Fisher, Proc. Cambridge Philos. Soc. 22, 700 (1925).

[28] S. López-Rosa, Doctoral thesis. information-theoretic measures of atomic and molecular systems, 2010, Dep. Física Atómica, Molecular y Nuclear. Univ. Granada, Spain.

[29] S. López-Rosa, R. O. Esquivel, J. C. Angulo, J. Antolín, J. S. Dehesa, and N. Flores-Gallegos, J. Chem. Theory Comput. 6, 145 (2009).

[30] R. O. Esquivel, N. Flores-Gallegos, C. Iuga, E. M. Carrera, J. C. Angulo, and J. Antolín, Theor. Chim. Acta (Berlin) 124, 445 (2009).

[31] P. Knowles and N. Handy, Comput. Phys. Commun. 54, 75 (1989).

[32] M. J. Frisch, G. W. Trucks, H. B. Schlegel, G. E. Scuseria, M. A. Robb, J. R. Cheeseman, G. Scalmani, V. Barone, B. Mennucci, G. A. Petersson, H. Nakatsuji, M. Caricato, X. Li, H. P. Hratchian, A. F. Izmaylov, J. Bloino, G. Zheng, J. L. Sonnenberg, M. Hada, M. Ehara, K. Toyota, R. Fukuda, J. Hasegawa, M. Ishida, T. Nakajima, Y. Honda, O. Kitao, H. Nakai, T. Vreven, J. A. Montgomery, Jr., J. E. Peralta, F. Ogliaro, M. Bearpark, J. J. Heyd, E. Brothers, K. N. Kudin, V. N. Staroverov, R. Kobayashi, J. Normand, K. Raghavachari, A. Rendell, J. C. Burant, S. S. Iyengar, J. Tomasi, M. Cossi, N. Rega, J. M. Millam, M. Klene, J. E. Knox, J. B. Cross, V. Bakken, C. Adamo, J. Jaramillo, R. Gomperts, R. E. Stratmann, O. Yazyev, A. J. Austin, R. Cammi, C. Pomelli, J. W. Ochterski, R. L. Martin, K. Morokuma, V. G. Zakrzewski, G. A. Voth, P. Salvador, J. J. Dannenberg, S. Dapprich, A. D. Daniels, . Farkas, J. B. Foresman, J. V. Ortiz, J. Cioslowski, and D. J. Fox, Gaussian 09 Revision D.01, Gaussian Inc. Wallingford CT 2009.

[33] A. S. de Meras, M.-B. Lepetit, and J.-P. Malrieu, Chem. Phys. Lett. 172, 163 (1990).

[34] C. W. Bauschlicher Jr and S. R. Langhoff, J. Chem. Phys. 89, 4246 (1988).

[35] T. A. Keith, Aimall (version 14.11.23), 2014, TK Gristmill Software, Overland Park KS, USA (aim.tkgristmill.com).

[36] P. Salvador and E. Ramos-Cordoba, Apost-3d program, 2012, Universitat de Girona (Spain).

[37] P. Salvador and E. Ramos-Cordoba, J. Chem. Phys. 139, 071103 (2013).

[38] E. Matito and F. Feixas, DMn program, 2009, University of Girona (Spain) and University of Szczecin (Poland).

[39] E. Matito, ESI-3D: Electron sharing indices program for 3D molecular space partitioning, 2014, Institute of Computational chemistry and Catalysis, University of Girona, Catalonia, Spain.

[40] S. Noury, X. Krokidis, F. Fuster, and B. Silvi, Topmod package, 1997.

[41] F. Feixas, E. Matito, M. Duran, M. Sola, and B. Silvi, J. Chem. Theory Comput. 6, 2736 (2010). 
[42] M. Rodríguez-Mayorga, RHO-OPS: Density operations., 2015, Institute of Computational Chemistry and Catalysis, University of Girona, Catalonia, Spain.

[43] S. C. Yang and W. C. Stwalley, ACS Symp. Series 179 (1982).

[44] R. S. Mulliken, Phys. Rev. 50, 1017 (1936).

[45] W. C. Stwalley and W. T. Zemke, J. Phys. Chem. Ref. Data 22, 87 (1993).

[46] B. Silvi and A. Savin, Nature 371, 683 (1994).

[47] V. Postils, M. Garcia-Borrás, M. Solà, J. M. Luis, and E. Matito, Chem. Commun. 51, 4865 (2015).

[48] D. L. Cooper, J. Chem. Phys. 80, 1961 (1984).

[49] E. Miliordos and A. Mavridis, J. Chem. Phys. 128, 144308 (2008).

[50] W. Heyndrickx, P. Salvador, P. Bultinck, M. Solà, and E. Matito, J. Comput. Chem. 32, 386 (2011).

[51] E. Matito, J. Poater, M. Solà, M. Duran, and P. Salvador, J. Phys. Chem. A 109, 9904 (2005).

[52] E. Ramos-Cordoba, E. Matito, P. Salvador, and I. Mayer, Phys. Chem. Chem. Phys. 14, 15291 (2012).

[53] V. Murg, F. Verstraete, R. Schneider, P. R. Nagy, and O. Legeza, J. Chem. Theory Comput. 11, 1027 (2015). 